\title{
Analysis of ultrasonic frequency response of surface attached fiber Bragg grating
}

\author{
Zhuoxuan Li, ${ }^{1,2}$ Li Pei, ${ }^{2, *}$ Bo Dong, ${ }^{1}$ Cheng Ma, ${ }^{1}$ and Anbo Wang ${ }^{1}$ \\ ${ }^{1}$ Center for Photonics Technology, Bradley Department of Electrical and Computer Engineering, Virginia Tech, \\ Blacksburg, Virginia 24061, USA \\ ${ }^{2}$ Institute of Lightwave Technology, Beijing Jiaotong University, Beijing 100044, China \\ ${ }^{*}$ Corresponding author: lipei@ bjtu.edu.cn
}

Received 14 March 2012; revised 25 May 2012; accepted 6 June 2012; posted 7 June 2012 (Doc. ID 164766); published 9 July 2012

\begin{abstract}
In recent years, fiber Bragg grating (FBG), for the well-known advantages over other fiber optic sensors, has attracted more attention in ultrasonic inspection for structure health monitoring (SHM). Spectrum shift of FBG to ultrasonic wave is caused by the refractive index profile changing along the FBG, which can be attributed to nonuniform perturbation caused by strain-optic and geometric effects of ultrasonic wave. Response of FBG to the above two effects was analyzed firstly by the $V-I$ transmission matrix model, showing high computing efficiency. Based on this model, spectra response of FBG under changing ultrasonic frequencies was simulated and discussed. In experiment, the system was able to detect a wideband ultrasonic wave ranging from 15 to $1380 \mathrm{kHz}$. These results would provide a guideline for an FBGbased acoustic detection system design in a specific ultrasonic frequency. (C) 2012 Optical Society of America

OCIS codes: $\quad 060.3735,230.1040,230.1480$.
\end{abstract}

\section{Introduction}

Ultrasonic inspection has been widely used in many scientific and industrial applications for nondestructive testing, such as aerospace, therapeutic applications, and automotive and other transportation sectors. In particular, when applied to structure health monitoring (SHM), wireless ultrasonic inspection has extensively been conducted for defect detection and thickness monitoring [1,2]. Conventional ultrasonic probes based on piezoelectricity were susceptible to technical problems such as electromagnetic interference and a lack of multiplexing capacity [3]. However, optical fiber sensors offer significant advantages over their electrical counterparts in terms of improved reliability and reduced physical profile. The most significant performance is their immunity to electromagnetic interference,

$1559-128 \mathrm{X} / 12 / 204709-06 \$ 15.00 / 0$

(C) 2012 Optical Society of America which enables applications under harsh environment [4]. In recent years, FBG has become an attractive candidate for ultrasonic wave detection, because it can be easily embedded in the structure of the body being monitored and is ideal for multiplexing $[5,6]$.

The principle of FBG acoustic detection lies in the fact that spectral shift would occur due to the acoustic-induced variations in the medium. The amplitude, frequency, phase, and polarization of the ultrasonic signal are influenced by the condition of the medium [7,8]. Tsuda [9] designed a Bragg wavelength-insensitive FBG vibration-sensing system employing a fiber ring laser that works at Bragg wavelength, and the intensity of light reflected from FBG varied due to vibration. This system has demonstrated the detection of vibrations at $7.5 \mathrm{~Hz}$. Fomitchov and Krishnaswamy [10] found that the $1.7 \mathrm{~mm}$ fiber Bragg grating (FBG) sensor was capable of detecting ultrasonic signals within the 0.5 to $5.0 \mathrm{MHz}$ frequency range; also the sensitivity of the sensor and its directional response were 
measured. Ultrasonic waves act on uniform FBG via strain-optic and geometric effects. Based on above two effects, Minardo et al. [11] modeled FBG as a layered and one-dimensional medium using the traditional transfer matrix method and numerically evaluated the responses of uniform and apodized FBGs to ultrasonic wave, but without experimental demonstration. In this paper, FBG response to an ultrasonic wave was analyzed by the $V-I$ transmission matrix method, showing the grating is not sensitive to high frequency when the ultrasonic wavelength is close to the grating length, which is consistent with those reported in [11]. Compared to the traditional transfer matrix method, the $V-I$ transmission matrix method can improve the computing efficiency with guaranteed accuracy. Experiments were carried out to demonstrate wideband ultrasonic wave detection ranging from 15 to $1380 \mathrm{kHz}$.

\section{Theoretical Analysis}

\section{A. Introduction of $V$-I Transmission Matrix Model}

Each fiber grating is composed of many layers, of which the number depends on the length of the segment and the distributing form of the refractive index. For nonuniform fiber grating, the calculation accuracy increases with the number of segments, however, at the expense of reduced computing efficiency. In this paper, for the uniform FBG we used, the segment length equals the grating length. Figure 1 shows incident field $E_{i}$ and reflected field $E_{r}$ propagating through the $m$ th layer and the $(m+1)$ th layer. The transmission matrix relates the two adjacent fields in the following way:

$$
\left[\begin{array}{c}
E_{m, i} \\
E_{m, r}
\end{array}\right]=A \cdot\left[\begin{array}{l}
E_{m+1, i} \\
E_{m+1, r}
\end{array}\right] .
$$

Matrix $A$ implies two physical processes: the transfer between two layers and the propagation through a lossless layer, respectively.

The $V-I$ transmission matrix model is often used to solve the transmission lines problem in the microwave signal processing area; however, it can also be introduced to the photonics area. The tangential electric field $V_{m}$ and magnetic field $I_{m}$ correspond to the electric field of the fiber in the following way:

$$
V_{m}=E_{m, i}+E_{m, r}, \quad I_{m}=\frac{1}{\Omega}\left(E_{m, i}-E_{m, r}\right),
$$

where $\Omega=120 \pi / n$ represents impedance of dielectric, and $n$ is the refractive index of the layer. After

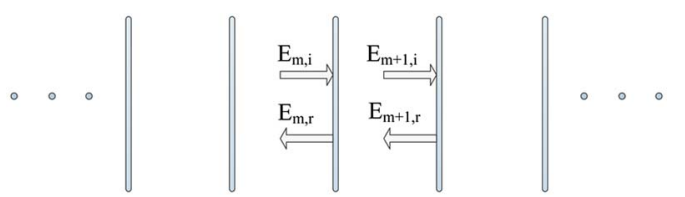

Fig. 1. (Color online) Light propagates through adjacent layers within one period of fiber grating. above transformation, in the $V-I$ model, the transfer matrix between two layers has turned into a unity matrix, and the propagation matrix is given as [12]

$$
V I_{P}=\left[\begin{array}{cc}
\cos \phi & j \Omega \sin \phi \\
j \frac{\sin \phi}{\Omega} & \cos \phi
\end{array}\right], \phi=\frac{2 \pi n}{\lambda} l,
$$

where $\phi$ is the phase of the grating layer, $\lambda$ is the optical wavelength, and $l$ is the length of one layer (half of the grating period). The integrated transmission matrix of the fiber grating can be expressed by multiplexing all the propagation matrices.

Coupled mode theory, as a usual way to get the spectra response of FBG, works accurately and swiftly [13]. Nevertheless, this method will be useless when FBG is immersed in an ultrasonic wave that produces a nonuniform perturbation. In such a case, FBG must be divided into a number of layers to represent the real case. In this regard, the $V-I$ transmission matrix model could satisfy this requirement well. The reflection spectra of a uniform FBG were solved by the $V-I$ transmission matrix and the traditional matrix method; the spectrum subtraction is plotted in Fig. 2. The FBG simulated was $3 \mathrm{~cm}$ in length. The two results are basically consistent with each other. The execution time for traditional method is $14.65 \mathrm{~s}$, as compared to $9.33 \mathrm{~s}$ for the $V-I$ transmission matrix method.

In this subsection, the possibility of saving matrix calculations by using the $V-I$ transmission matrix model has been pointed out. It is in each layer that only one matrix is operated, and in addition, the $V-I$ model provides a viable means to calculate more complex FBG structures [14].

\section{B. Strain-Optic and Geometric Effects Impinging on FBG}

FBG is produced by exposing an optical fiber to a spatially varying pattern of ultraviolet intensity, resulting in a sinusoidal perturbation to the effective refractive index of the guided modes. In the $V-I$ transmission matrix model, FBG is divided into a large number of layers by half of its period, and

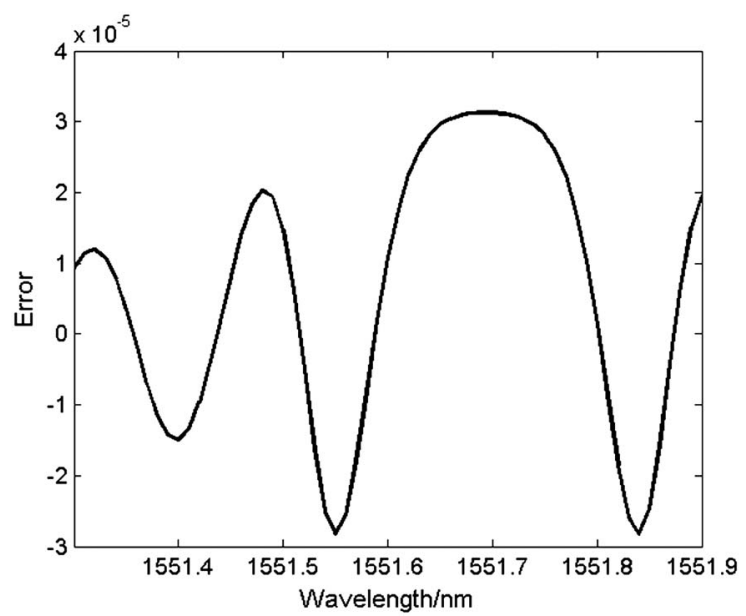

Fig. 2. Error between reflection spectrum solved by $V-I$ transmission matrix method and traditional transmission matrix method. 
we approximate the sinusoidal function $n(z)$ by a periodic square function $n^{\prime}(z)$, of which the amplitude is $d n$, shown in Fig. 3, expressed as follows:

$$
\begin{aligned}
n(z) & =N+d n+d n \sin (2 \pi z / \Lambda), \\
n^{\prime}(z) & \approx N+d n+\frac{4}{\pi} d n \sin (2 \pi z / \Lambda),
\end{aligned}
$$

where $N$ is the effective refractive index of the guided modes in the absence of grating. So we could apply the fundamental term of the Fourier series of the periodic square function instead of the sinusoidal function; as we can see from Eq. (4), a factor of $\pi / 4$ is required to make the approximation from the square distribution to the sinusoidal one.

An ultrasonic wave causes a refractive index change via the strain-optic effect. Supposing it brings no shear strain and the fiber is isotropic and homogeneous, the refractive index relates to the ultrasonic effect as follows [4]:

$$
\begin{aligned}
n^{\prime}\left(z^{\prime}, t\right)= & n(z)+\Delta n\left(z^{\prime}, t\right)=\frac{\pi}{4} n^{\prime}(z)+\left(1-\frac{\pi}{4}\right)(N+d n), \\
& -\frac{1}{2} N^{3}\left[\varepsilon_{\mathrm{US}}(1-\sigma) p_{12}-\sigma \varepsilon_{\mathrm{US}} p_{11}\right] \cdot \varepsilon_{\mathrm{US}} \\
& \cos \left(k_{\mathrm{US}} z^{\prime}-w_{\mathrm{US}} t\right),
\end{aligned}
$$

where $\varepsilon_{\mathrm{US}}$ is the amplitude of the ultrasonic wave, $\sigma$ is the Poisson's ratio, $p_{11}$ and $p_{12}$ are the strain-optic coefficients, $k_{\mathrm{US}}=2 \pi / \lambda_{\mathrm{US}}$ is the wave-number at the ultrasonic wavelength $\lambda_{\mathrm{US}}$, and $w_{\mathrm{US}}$ is its angular frequency. $z^{\prime}$ is defined by

$$
z^{\prime}=z+\varepsilon_{\mathrm{US}} \frac{\lambda_{\mathrm{US}}}{2 \pi} \sin \left(\frac{2 \pi}{\lambda_{\mathrm{US}}} z-w_{\mathrm{US}} t\right) .
$$

Ultrasonic wave induced Geometric deformation along the FBG can be described as a series of expansions and compressions, yielding length change of each layer with time dependent $\Delta l(z, t)$. So the layer length relating to the ultrasonic effect could be written as

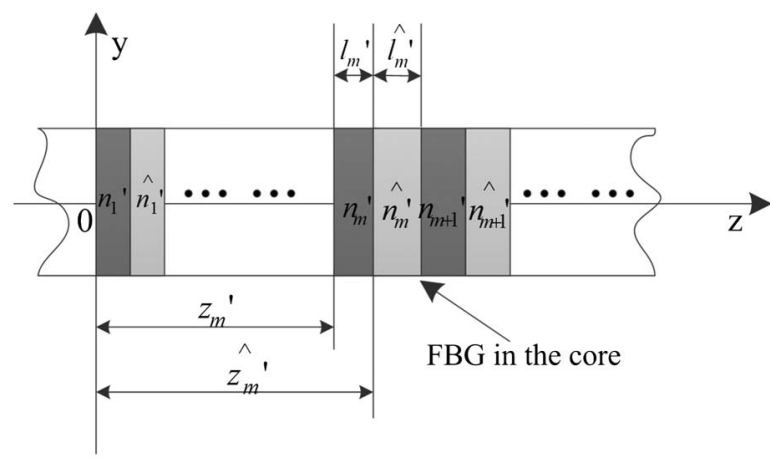

Fig. 3. The refractive index distribution of FBG before ultrasonic wave interacting with. $l^{\prime}\left(z^{\prime}, t\right)=l+\Delta l\left(z^{\prime}, t\right)=\frac{\Lambda}{2}\left[1+\varepsilon_{\mathrm{US}} \cos \left(k_{\mathrm{US}} z^{\prime}-w_{\mathrm{US}} t\right)\right]$.

Within each period, the parameters of latter layer were denoted as $\hat{n}_{m}^{\prime}, \hat{l}_{m}^{\prime}$, and $\hat{z}_{m}^{\prime}$ to account for nonuniform refractive index distribution, as shown in Fig. $\underline{4}$, where $m$ denotes the period index of the FBG. Now the $m$ th period $V-I$ matrix expression is attainable by use of Eq. (3), after ultrasonic wave impinging on the FBG:

$$
\begin{aligned}
V I_{m} & =\left[\begin{array}{cc}
\cos \phi & j \Omega \sin \phi \\
j \frac{\sin \phi}{\Omega} & \cos \phi
\end{array}\right] \times\left[\begin{array}{cc}
\cos \hat{\phi} & j \hat{\Omega} \sin \hat{\phi} \\
j \frac{\sin \hat{\phi}}{\hat{\Omega}} & \cos \hat{\phi}
\end{array}\right], \\
\phi & =\frac{2 \pi n_{m}^{\prime}}{\lambda} l_{m}^{\prime}, \quad \Omega=\frac{120 \pi}{n_{m}^{\prime}}, \\
\hat{\phi} & =\frac{2 \pi \hat{n}_{m}^{\prime}}{\lambda} \hat{l}_{m}^{\prime}, \quad \hat{\Omega}=\frac{120 \pi}{\hat{n}_{m}^{\prime}} .
\end{aligned}
$$

By multiplexing all of the periodic matrices together, the transmission matrix of the whole FBG can be written as

$$
V I=\left[\begin{array}{ll}
A & B \\
C & D
\end{array}\right]=\prod_{m=1}^{M} V I_{m} .
$$

Therefore, we could get the reflectivity of the FBG at the wavelength of $\lambda$ with the following equation [12]:

$$
R=|r|^{2}=\left(\frac{A+B / \hat{\Omega}-C \Omega-D \Omega / \hat{\Omega}}{A+B / \hat{\Omega}+C \Omega+D \Omega / \hat{\Omega}}\right)^{2},
$$

where $r$ is the reflection coefficient.

\section{Numerical Simulation}

The strongest interaction or mode-coupling occurs at the Bragg wavelength. Figure 5 shows the original reflected spectrum of an FBG, the spectra under the strain-optic and geometric effects, and the overall spectrum resulting from the cooperation of the two effects. In Fig. 5, the FBG length $L$ is $1.1 \mathrm{~cm}$, and

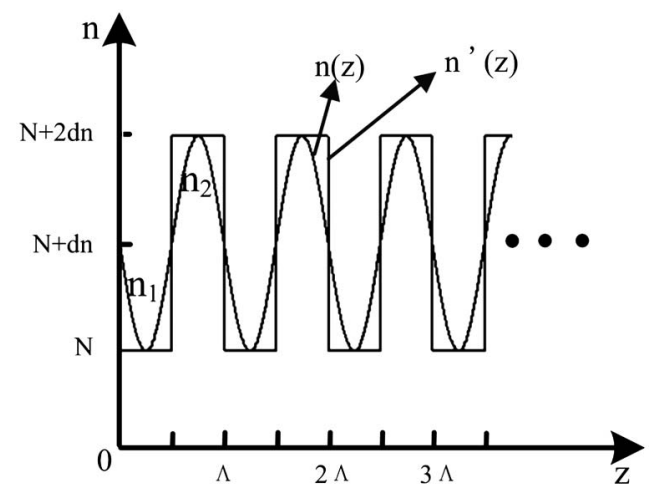

Fig. 4. Schematic diagram of the FBG composed of multilayers after interacting with ultrasonic wave. 


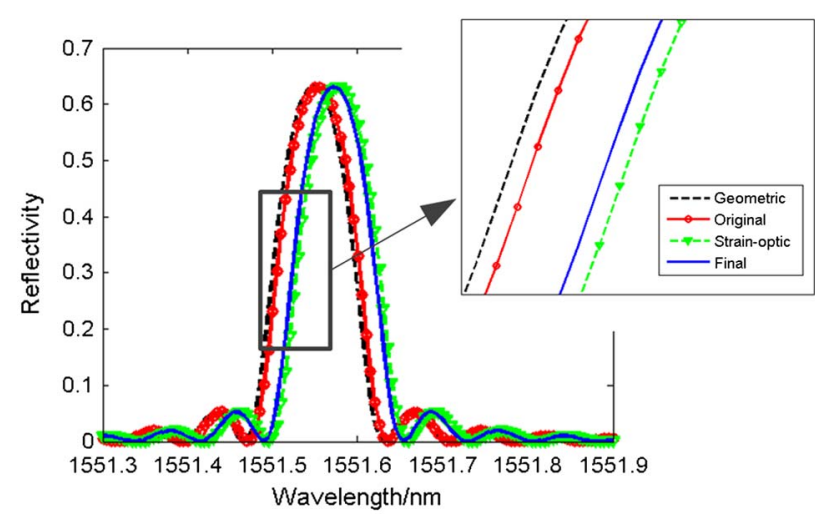

Fig. 5. (Color online) Reflected spectrum shift of FBG under strain-optic and geometric effects.

the full width at half maximum (FWHM) bandwidth is $0.13 \mathrm{~nm}$. The ultrasonic wave oscillates in a direction parallel to the grating axis, with an amplitude of $15 \mu \varepsilon$ and a frequency of $100 \mathrm{kHz}$.

Distortion of the FBG spectra may occur if ultrasonically induced grating nonuniformity takes place. The spectral responses of FBG to the ultrasonic wave with different wavelengths at $t=0$ are shown in Fig. 6 . The amplitude of the ultrasonic wave is set as $100 \mu \varepsilon$, and the parameters of FBG are set identically to the case in Fig. 5 . Figure 6 indicates that the FBG becomes irresponsive when the ultrasonic wavelength $\lambda_{\text {US }}$ is considerably small compared with the length of FBG. The spectral distortion appears when $\lambda_{\text {US }}$ and $L$ are in comparable order, and becomes trivial when $\lambda_{\mathrm{US}}$ is sufficiently larger than $L$, which could be explained by Eqs. (5) and (7): In the case of $\lambda_{\text {US }}$ being much greater than $L$, the cosine modulation term is equal to 1 , which means the ultrasonic wave induces uniform change along the length of the FBG.

Figure 7 shows the maximum amplitude change of the reflectivity of FBG at FWHM. The simulation result provides a guideline for FBG-based acoustic detection system design given the ultrasonic wavelength.

\section{Experimental Arrangement}

In Figure 8 , the left picture shows the experimental arrangement of the FBG-based ultrasonic sensor. A wideband PZT (lead zirconate titanate) transducer is connected to an acoustic emission preamplifier (2/4/6-C, Physical Acoustics Corporation). A $15 \mathrm{MHz}$ waveform generator (33120A, Agilent) produces modulated sinusoidal pulses with an amplitude of $1-5 \mathrm{~V}$, propagating through a $5 \mathrm{~mm}$-thick aluminum plate. A tunable laser (TUNICS-BT) with the linewidth of $100 \mathrm{kHz}\left(8 \times 10^{-4} \mathrm{pm}\right)$ and the wavelength resolution of $1 \mathrm{pm}$ was tuned to the FWHM point of FBG to obtain the optimum sensitivity, and the output power is set to $3 \mathrm{dBm}$. The FBG is fabricated with parameters representing the numerical simulation. A circulator guides the reflected light from the FBG to a photodetector (Thorlabs FPD310 $1.8 \mathrm{GHz}$, $\left.5 \times 10^{-4} \mathrm{~V} / \mathrm{W}\right)$. The FBG and the PZT are surface-
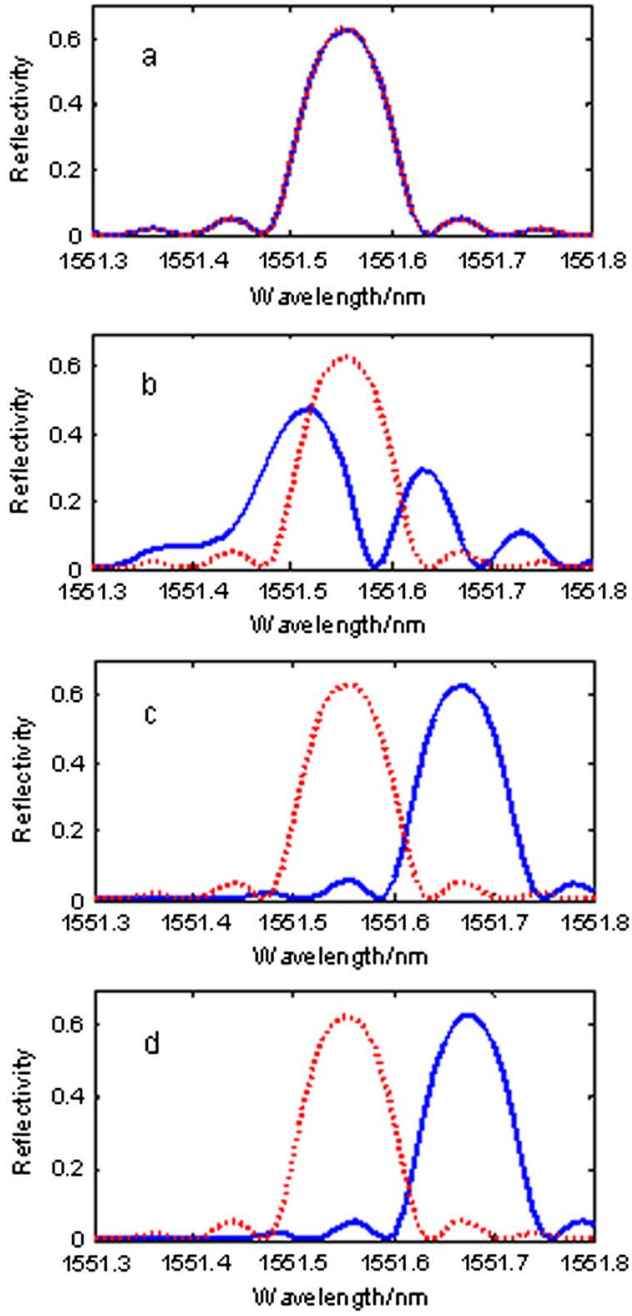

Fig. 6. (Color online) Original FBG spectra (dotted lines) and spectra under influences of ultrasonic waves (solid lines) calculated for (a) $\lambda_{\mathrm{US}} / L=0.1$; (b) $\lambda_{\mathrm{US}} / L=1$; (c) $\lambda_{\mathrm{US}} / L=10$; (d) $\lambda_{\mathrm{US}} / L=100$.

attached to an aluminum plate, with a distance of $30 \mathrm{~cm}$. The oscilloscope (LeCroy Wavepro7Zi) with an embedded computer monitors the frequency

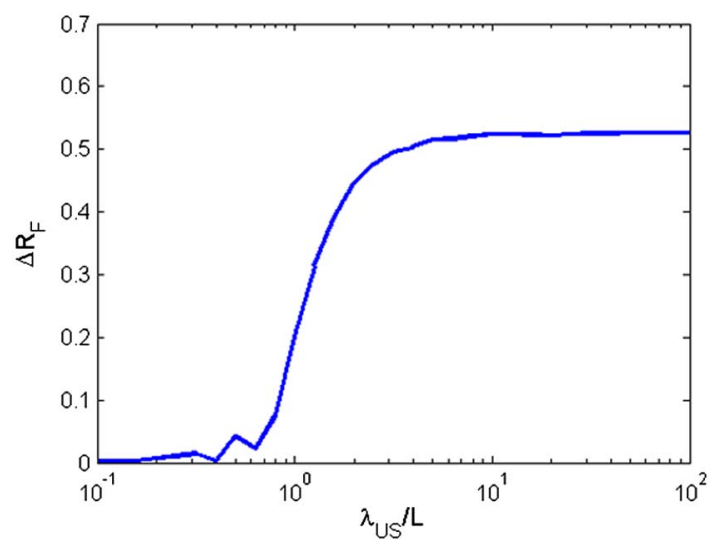

Fig. 7. (Color online) Maximum amplitude change of reflectivity at FWHM $\Delta R_{F}$ versus the ratio $\lambda_{\text {US }} / L$. 


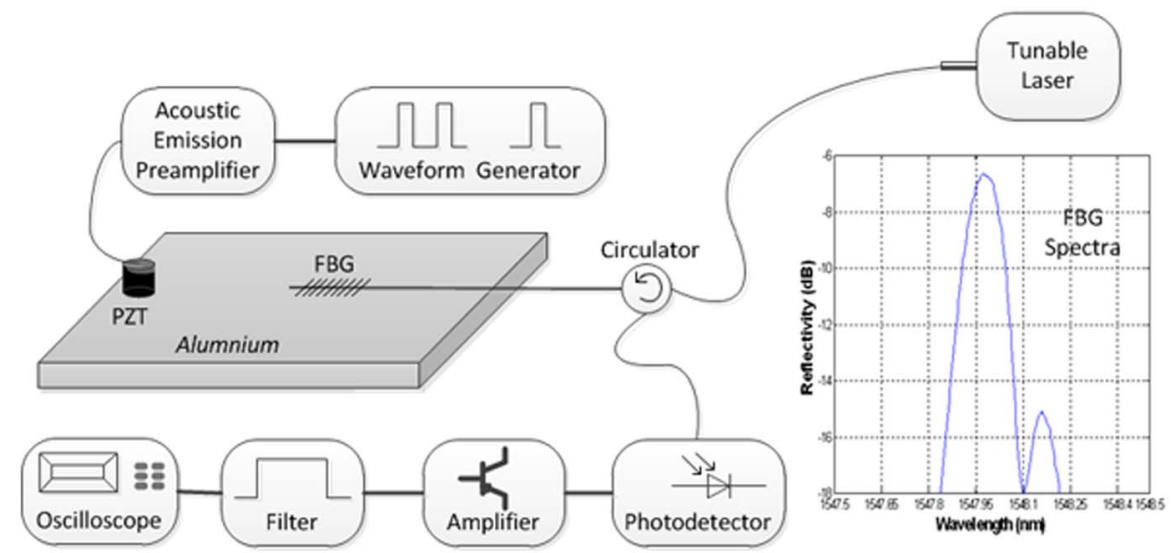

Fig. 8. (Color online) Schematic diagram of the acoustic emission and detection scheme (left) and the spectrum of FBG used in the experiment (right).

response of the FBG by recording the intensity of the acoustic signal. The right picture shows the reflected spectrum of a $1.1 \mathrm{~cm}$-long fabricated FBG free of acoustic wave.

Figure 9 shows the FBG response, after a series of amplification and filtering, to an ultrasonic wave excited by an eight-cycle burst acoustic signal with frequency of $90 \mathrm{kHz}$. The smoothed and broadened response waveform can be attributed to the reflections of the acoustic waves from boundaries of the plate and acoustic mode conversions. Betz et al. [15] described the signal processing techniques used for damage detection. They applied the Hilbert transform and the continuous wavelet transform to relate the changes in the Lamb wave signals to the occurrence of damage. The interpretation of the received ultrasonic signals is one part of our future work.

In this experiment, the wavelength of the ultrasonic wave was tuned continuously by controlling the feeding waveform generator. In order to characterize the FBG frequency response, the amplitude of the ultrasonic wave generated by the PZT maintained

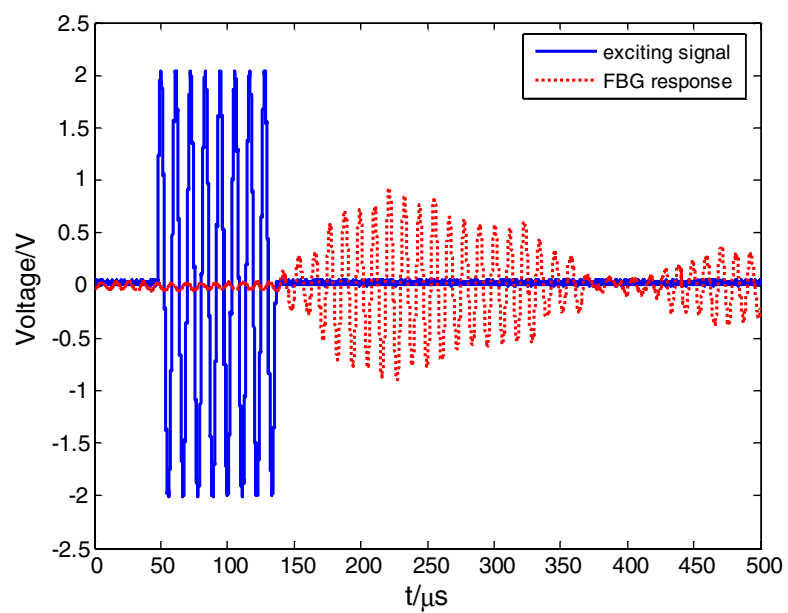

Fig. 9. (Color online) The PZT excitation waveform (solid line) and the FBG response (dotted line). fixed during frequency scanning. Then the output wavelength of the laser was set at the left FWHM point of the FBG (1547.9 nm). The acoustic frequency range (from 15 to $1380 \mathrm{kHz}$ ) was translated to a wavelength range using the following equation:

$$
\lambda_{\mathrm{US}}=\nu / f
$$

where $\nu$ is the velocity of ultrasonic wave propagating through aluminum. Figure 10 shows the normalized FBG response with respect to $\lambda_{\mathrm{US}} / L$, where $L=1.1 \mathrm{~cm}$. The result in Fig. 10 is consistent with the numerical result in Fig. 7 , except two abnormal points at $\lambda_{\mathrm{US}} / L=1.26$ and $\lambda_{\mathrm{US}} / L=3.98$, corresponding to ultrasonic frequencies of 116 and $370 \mathrm{kHz}$, respectively. This was attributed to the mechanical resonance of the FBG. The resonant acoustic frequency decreased when a longer FBG was applied. We also noticed that the resonant frequency was shifted when the host material had been replaced. That is because the acoustic velocity will change when different host materials are applied, and the velocity is related to the resonant frequency.

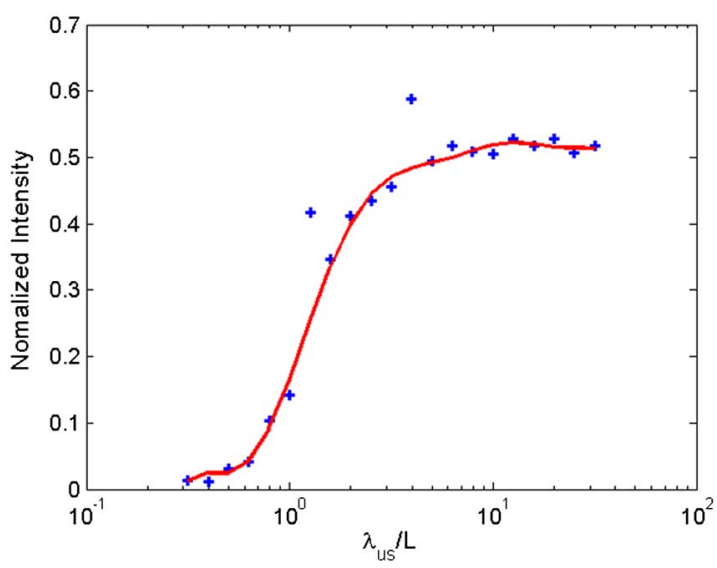

Fig. 10. (Color online) Normalized optical signal strength plotted with respect to the ratio $\lambda_{\mathrm{US}} / L$, with $L$ being the FBG length. 


\section{Conclusion}

Response of FBG to an ultrasonic wave was investigated theoretically and experimentally. In part A of the theoretical analysis, the $V-I$ transmission matrix model was introduced in an ultrasonic area. This method could improve computing efficiency because half of the matrices involved are unity matrices. In part B, with an ultrasonic wave impinging on FBG by strain-optic and geometric effects, the length of each layer and the refractive index distribution along the grating were obtained based on this model. In numerical simulation part, the FBG response at $t=0$ with four different ratios of $\lambda_{\mathrm{US}} / L$ were given, showing how this significant parameter affects the performance of FBG reflection spectra. Results from tuning the laser showed that the linear relation between acoustic pressure and signal intensity, and the maximum signal strength, could be achieved at the FWHM point of FBG reflected spectra. Thus, the maximum amplitude change of reflectivity $\Delta R_{F}$ as a function of the ratio provided a viable means for FBG acoustic detection system design.

A FBG-based ultrasonic wave inspection platform was built to experimentally demonstrate the theoretical finding. The frequency response of the FBG sensor was characterized in the range of 15 to $1380 \mathrm{kHz}$, and the measurement results agreed well with the theoretical analysis. This concluded that the frequency response of the FBG is primarily determined by the structural and optical nonuniformity along the FBG as dictated by the ultrasonic periodicity via the strain-optic and geometric effects, as elaborated by the $V-I$ transmission matrix model. The theoretical and experimental evidences provided herein shed light on optimal system design for ultrasonic nondestructive testing based on FBG sensors.

\section{References}

1. D. Clorennec, C. Prada, and D. Royer, "Laser ultrasonic inspection of plates using zero-group velocity lamb modes,"
IEEE Trans. Ultrason. Ferroelectr. Freq. Control 57, 11251132 (2010).

2. C. J. Lane, A. K. Dunhill, B. W. Drinkwater, and P. D. Wilcox, "The inspection of anisotropic single-crystal components using a 2-D ultrasonic array," IEEE Trans. Ultrason. Ferroelectr. Freq. Control 57, 2742-2752 (2010).

3. N. E. Fisher, D. J. Webb, C. N. Pannell, D. A. Jackson, L. R. Gavrilov, J. W. Hand, L. Zhang, and I. Bennion, "Ultrasonic field and temperature sensor based on short in-fibre Bragg gratings," Electron. Lett. 34, 1139-1140 (1998).

4. G. Wild and S. Hinckley, "Acousto-ultrasonic optical fiber sensors: overview and state-of-the-art," IEEE Sens. J. 8, 1184-1193 (2008).

5. S. Campopiano, A. Cutolo, A. Cusano, M. Giordano, G. Parente, G. Lanza, and A. Laudati, "Underwater acoustic sensors based on fiber Bragg gratings," Sensors 9, 4446-4454 (2009).

6. A. Rosenthal, D. Razansky, and V. Ntziachristos, "Highsensitivity compact ultrasonic detector based on a pi-phaseshifted fiber Bragg grating," Opt. Lett. 36, 1833-1835 (2011).

7. C. Betz, G. Thursby, B. Culshaw, and W. J. Staszewski, "Acousto-ultrasonic sensing using fiber Bragg gratings," Smart Materials and Structures 12, 122-128 (2003).

8. H. P. Loock, W. S. Hopkins, C. M. Blair, R. Resendes, J. Saari, and N. R. Trefiak, "Recording the sound of musical instruments with FBGs: the photonic pickup," Appl. Opt. 48, 2735-2741 (2009).

9. H. Tsuda, "Fiber Bragg grating vibration-sensing system, insensitive to Bragg wavelength and employing fiber ring laser," Opt. Lett. 35, 2349-2351 (2010).

10. P. Fomitchov and S. Krishnaswamy, "Response of a fiber Bragg grating ultrasonic sensor," Opt. Eng. 42, 956-963 (2003).

11. A. Minardo, A. Cusano, R. Bernini, L. Zeni, and M. Giordano, "Response of fiber Bragg grating to longitudinal ultrasonic waves," IEEE Trans. Ultrason. Ferroelectr. Freq. Control 52, 304-312 (2005).

12. J. Capmany, M. A. Muriel, S. Sales, J. J. Rubio, and D. Pastor, "Microwave $V-I$ transmission matrix formalism for the analysis of photonic circuits: application to fiber Bragg gratings," J. Lightwave Technol. 21, 3125-3134 (2003).

13. M. McCall, "On the application of coupled mode theory for modeling fiber Bragg gratings," J. Lightwave Technol. 18, 236-242 (2000).

14. Z. X. Li, L. Pei, C. H. Qi, W. J. Peng, T. G. Ning, R. F. Zhao, and S. Gao, "Fiber grating Fabry-Perot cavity studied by $V$ - $I$ transmission matrix method," Acta Phys. Sin. 59, 8615-8624 (2010).

15. D. C. Betz, W. J. Staszewski, G. Thursby, and B. Culshaw, "Structural damage identification using multifunctional Bragg grating sensors: II damage detection results and analysis," Smart Materials and Structures 15, 1313-1322 (2006). 